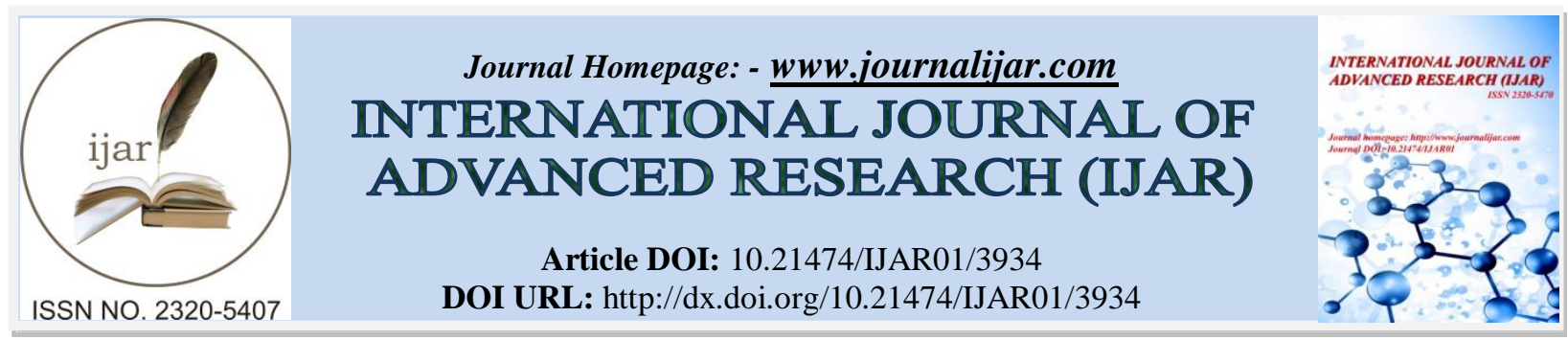

RESEARCH ARTICLE

\title{
SMART DENTAL MATERIALS: A REVIEW OF THE CURRENT LITERATURE.
}

\section{Dr. Riyas Ahamed K ${ }^{1}$, Dr. Prathap M.S ${ }^{2}$ and Dr. K Harish S Shetty ${ }^{3}$.}

1. Post graduate student, Department of Conservative Dentistry and Endodontics, Yenepoya Dental college Deralakatte-575018.

2. Professor \& guide, Department of Conservative Dentistry and Endodontics, Yenepoya Dental College Deralakatte-575018.

3. Senior professor \& Head of the Department, Department of Conservative Dentistry and Endodontics, Yenepoya Dental College Deralakatte-575018.

\section{Manuscript Info}

Manuscript History

Received: 14 February 2017

Published: April 2017
Final Accepted: 16 March 2017

\begin{abstract}
Dental materials are stable and have greater durability if they do not react with the environment and remain passive. At the same time, it is also expected that these materials will be well accepted and will cause neither harm nor injury. This is an entirely negative approach to material tolerance and biocompatibility. This outlook hides the possibility through which positive gains can be achieved by using materials that behave in a more dynamic fashion in the environment in which they are placed. The current dental materials are improvised. The use of smart materials has brought a great revolution in dentistry. The smart behaviour of materials occur when it sense some stimulus from surrounding environment and reacts to it in an intelligent way to do a specific function. These materials can be altered in a controlled manner by various stimuli such as stress, temperature, moisture, $\mathrm{pH}$ and electric or magnetic field. The key feature of smart behaviour includes its ability to return to it's original state after the stimulus is removed. Some of the smart materials used are resin modified glass ionomers, amorphous calcium phosphate releasing pit and fissure sealants, smart composites, smart ceramics, smart antimicrobial peptide, smart coating on implant, orthodontic shape-memory alloys, smart impression materials, smart sutures, smart burs, smart fibers, smart endodontic files etc. These materials have revolutionized dentistry and hence the beginning of new chapter in Biosmart Dentistry
\end{abstract}

Copy Right, IJAR, 2017,. All rights reserved.

\section{Introduction:-}

Mostly all the materials were designed to act in a passive way with no interaction with the oral environment. This was to ensure that materials could survive longer and allowing them to be used for longer periods. Then, it was realized that some materials were able to act in an 'active' way. These materials can positively react in an oral environment. Materials showing this type of behaviour are considered as "Smart materials". Today the most promising technology for lifetime efficacy and improved reliability include the use of "bioactive" smart materials. McCabe Zrinyi defined smart materials as "Materials that are able to be altered by stimuli and transform back into the original state after removing the stimuli". ${ }^{1}$ The stimuli can be derived from temperature, $\mathrm{pH}$, moisture, stress,

Corresponding Author:- Riyas Ahamed K.

Address:- Post graduate student, Department of Conservative Dentistry and Endodontics, Yenepoya 
electricity, chemical or biomedical agents and magnetic fields. A key feature of smart behaviour includes the ability to return to the original state after the stimulus has been removed. Due to the interesting behaviour of smart materials, scientists are encouraged to apply them in various fields, mostly into biomedical science and dentistry. The different types of smart materials used in the field of dentistry are piezoelectric materials, shape memory alloys or shape memory polymers, $\mathrm{pH}$ sensitive polymers, polymer gels and the recent ones have shown their own smart behaviour. Smart materials are highly responsive and have a great capacity to sense and respond to any environmental changes. Hence these materials are also known as "Responsive Materials". Smart materials can happen by chance or they can be designed to incorporate smartness in them. ${ }^{2}$

\section{Properties of Smart Materials:-}

Smart materials sense changes in the environment around them and respond in a predictable manner. In general, their properties are:

* Piezoelectric ${ }^{3}$ - when a mechanical stress is applied, an electric current is generated.

- Shape memory ${ }^{4,5}$ - after deformation these materials can remember their original shape and return back to its original shape when heated.

* Thermochromic ${ }^{6}$ - these materials change colour in response to changes in temperature.

* Photochromic ${ }^{6}$ - these materials change colour in response to changes in light conditions.

* Magnetorheological ${ }^{6}$ - these are fluid materials become solid when placed in a magnetic field.

* PH sensitive $\mathrm{e}^{5}$ - materials which swell/collapse when the $\mathrm{pH}$ of the surrounding media changes.

- Bio film formation ${ }^{7}$ - presence of bio film on the surface of material alters the interaction of the surface with the environment.

\section{Classification of Smart Materials:-}

Passive Smart Materials: They sense the external change and react to it without external control. They also possess self - repairing property. ${ }^{8,9}$

- GIC

- Resin Modified GIC

- Compomer

- Dental Composites

Active Smart Materials:-

Active materials sense change in the environment and respond to them. Utilize a feedback loop to enable them to functions as a cognitive response through a controlled mechanism or system. ${ }^{8,9}$

Followings are the various types of smart materials in the different branches of the dentistry:Restorative Dentistry:-

- Smart GIC

- Smart composites

- Smart Prep Burs

- $\quad$ Smart bonding system

\section{Prosthetic Dentistry:-}

- Smart ceramics

- Smart impression materials

\section{Orthodontics:-}

- Shape memory alloys.

- Smart orthodontic adhesive

\section{Pediatric and Preventive Dentistry:-}

- Fluoride releasing pit and fissure sealants

- ACP releasing pits and fissure sealants.

\section{Endodontics:-}

- Niti Rotary Instruments. 
- Smartseal obturation system

Laser Dentistry:-

- Smart Fibers.

Periodontics:-

- Smart antimicrobial peptide.

Implant Dentistry:-

- Smart coatings on implant.

Oral surgery:-

- Smart sutures

Amorphous Calcium Phosphate (ACP):-

Aaron S. Posner has described ACP for first time in 1963. At neutral or high pH, ACP remains in its original form. But at or below 5.8 (critical pH), demineralisation of tooth surface will take place. As a result ACP gets converted to crystalline Hydroxyapatite (HAP), thus replacing the HAP crystal lost by acid. These free ions will get merged and form gel-like structure within seconds. Within 2 minutes this gel-like structure releases calcium and phosphate ions. These calcium and phosphate ions neutralizes and buffers the $\mathrm{pH}$, which justify its use in bone repair materials, smart coated implants, dental varnish (Enamel Pro varnish), chewing gums, dentifrices, mouth washes, dental cements and orthodontic adhesive (Aegis Ortho),pit and fissure sealants, composites and dental adhesives. ${ }^{2,9}$

Casein Phosphopeptide (CPP):-

A milk derivative, in combination with ACP (CPP-ACP complex) is used for the remineralisation of incipient white spot lesions in some dentifrices under the name ReCaldent. ${ }^{6}$ It is marketed as GC tooth mousse plus ${ }^{\circledR}$ - (The University of Melbourne, Victoria, Australia) and a new GIC containing 3\%(w/w)CPP-ACP (Fuji VII EP)

\section{Smart GIC:-}

Smart behaviour was seen for the first time in GIC by Davidson. On intake of hot or cold food and fluids, these restorative materials may show thermal expansion or contraction in response to thermal stimuli. The mismatch of thermal expansion and contraction between a restoration and the tooth structure may result in stresses at the interface, and this may lead to microleakage. In dry condition, these materials showed a marked contraction when heated above $50^{0 \mathrm{c}}$. The explanation for this behaviour is that the expected expansion on heating is compensated by fluid flow to the surface of the material to cause a balancing of the dimensional changes. On cooling, this process is reversed. In dry condition, the rapid loss of water on heating results in the observed contraction. This behaviour is akin to that of human dentin where very little dimensional change is observed on heating in wet conditions and a marked contraction is noted in dry conditions. Both results can be explained by flow of fluids in the dentinal tubules. Hence glass ionomer materials can be said to be mimicking the behaviour of human dentin through a type of smart behaviour. Hence, GICs are described as "smart materials" with respect to their thermal behaviour. ${ }^{7,10}$

Additional smart behaviour of GIC is Fluoride release which helps in caries prevention. In the case of GICs, the fluoride release rate can become negligible within a week. However, this problem can be tackled. There is evidence that the fluoride released from salt phases can be replaced when the material is bathed in a high concentration of fluoride as may occur in a toothpaste or mouthwash. This long term fluoride release is more important than the initial 'burst' for a short time. As the material becomes depleted of its inherent fluoride it is interesting that the 'spikes' of fluoride after recharging appear to increase slightly with age. This implies that the more inherent fluoride lost, the greater is its capacity for uptake through recharging. Other studies have shown that the rates of fluoride release and recharging are temperature sensitive. Hence, a faster recharging can be attained by using warm fluoride containing solutions and this can generate a more sustained release at mouth temperature. These smart ionomer mimic the behaviour of human dentin. Resin modified glass ionomer cement, compomer or giomer all exhibit these smart characteristics. ${ }^{11,12}$ Ex: GC Fuji IX GP EXTRA (Zahnfabrik Bad Sackingen, Germany) 


\section{Smart Ceramics:-}

Zirconia is a polycrystalline ceramic that do not contain glass. All of its atoms are packed into regular crystalline arrays through which it is much more difficult to drive a crack than it is through atoms in the less dense and irregular network found in glasses. Hence, polycrystalline ceramics generally are much tougher and stronger than glass based ceramics. Well-fitting prosthesis made from polycrystalline ceramics were not practical before the availability of computer-aided manufacturing. In 1995, the first "all ceramic teeth bridge" was fabricated at ETH Zurich based on a process that enabled direct machining of bridges.

Pure zirconia at room temperature has a monoclinic crystalline structure and at firing temperature zirconia has a tetragonal crystalline structure. On cooling from firing temperature, it results in $4.4 \%$ increase in volume than at firing temperature (tetragonal crystalline structure). In the late 1980s, ceramic engineers learned to stabilize the tetragonal form at room temperature by adding small amounts of calcium and later yttrium or cerium.

Although stabilized at room temperature, the tetragonal form is "metastable," meaning that the trapped energy still exists within the material to drive it back to the monoclinic state, which can be induced by external influence like tension or temperature. A highly localized stress ahead of a propagating crack is sufficient to trigger grains of ceramic to transform in the vicinity of that crack tip. In this case, the $4.4 \%$ volume increase becomes beneficial, essentially altering material conditions around the crack tip, shielding it from the outside world. The result is that a compressive or crack closure stress is produced which slows down or stops the crack. This crystallographic transformation in response to stress makes zirconia a smart material. ${ }^{13,14,15}$ Ex: Cercon Zirconium $\mathrm{S}$ mart Ceramic System

\section{Smart Composites:-}

Smart composite contains Amorphous Calcium Phosphate. ACP at neutral or high pH remains ACP. When low $\mathrm{pH}$ values i.e., at or below 5.8 occurs during a carious attack, ACP converts in to HAP and precipitates, thus replacing the HAP lost to the acid. So when the $\mathrm{pH}$ level in the mouth drops below 5.8, these ions merge within seconds to form a gel. In less than 2 minutes, the gel becomes amorphous crystals, resulting in calcium and phosphate ions. ${ }^{16,17}$

Ariston $\mathrm{pH}$ control - introduced by Ivoclar-Vivadent. It is a light-activated alkaline, nano filled glass restorative material. It releases calcium, fluoride and hydroxyl ions when intraoral $\mathrm{pH}$ values drop below the critical $\mathrm{pH}$ of 5.5 and counteracts the demineralization of the tooth surface and also aids in remineralisation. The material can be adequately cured in bulk thickness up to $4 \mathrm{~mm}$. It is recommended for the restoration of class 1 and class 2 lesions in both primary and permanent teeth. ${ }^{2}$

Self-repairing or self-healing composite - This is an epoxy system which contained resin filled microcapsules. If a crack occurs in the epoxy composite material, some of the microcapsules are destroyed near the crack to release the resin. The resin subsequently fills the crack and reacts with a Grubbs catalyst dispersed in the epoxy composite, resulting in polymerization of the resin and repair of the crack. Similar systems were demonstrated to have a significantly longer duty cycle under mechanical stress in situ compared to similar systems with the self-repair. $^{18,19,20}$

\section{Shape Memory Alloys:-}

Shape memory alloys (SMA) are the metals with the ability to recover the original shape/length when subjected to the thermo- mechanical load. These alloys show properties like super elasticity, shape memory, good resistance to fatigue and wear and relatively good biocompatibility. ${ }^{1,5}$ Ex: Nickel -Titianium

\section{Nickel Titanium Alloy:-}

Greniger and Mooradian in 1938, first noticed shape memory property of copper- zinc and copper tin alloys. ${ }^{2}$ Nickel-Titanium was developed by the Naval Ordinance Laboratory (NOL) in Silver Springs, Maryland by Buehler et al. 50 years ago. Nitinol basically exist in two phases. The low temperature phase is called the martensitic or daughter phase (a body centred cubic lattice), and the high temperature phase is called the austenitic or parent phase (hexagonal lattice).This lattice organisation can be altered either by stress or temperature. ${ }^{1, t}$ 
In endodontics, Walia et al. in 1988 introduced $\mathrm{Ni}-\mathrm{Ti}$ to Endodontics. 55wt\% $\mathrm{Ni}$ and $45 \mathrm{wt} \% \mathrm{Ti}$ are commonly used, referred as "55NiTiNOL". The super-elasticity of NiTi rotary endodontic instruments provide improved access to curved root canals during the chemomechanical preparation with a lesser lateral force being exerted. It allows more canal centred preparations with reduced incidence of canal transportation and aberrations. Nitinol shows stress-induced thermoelastic transformation. Generally, it is in an austenitic crystalline phase that gets converted to a martensitic structure on stressing at a constant temperature. In this martensitic phase, only a light force is sufficient for bending. By relieving the stress, original shape is regained in its austenitic phase. ${ }^{2,7,10}$

In orthodontics, $\mathrm{NiTi}$ arch wires are used instead of stainless steel on account of their limited flexibility and tensile properties. NiTi wires, because of their super elasticity and shape memory, apply continuous gentle forces on the teeth, for a long period with in physiological range. ${ }^{2}$

\section{Smartseal Obturation System:-}

Obturation of root canals should prevent reinfection of the canal space and ultimately prevent periradicular disease. This objective may be achieved by three-dimensional filling of the instrumented canal, accessory canals, and dead spaces.

The smartseal obturation system is a point-and-paste root canal filling technique that consists of premade, hydrophilic endodontic points and an accompanying sealer. The deformable point is available in different tip sizes and tapers and is designed to expand laterally without increase in length by absorbing residual water from the instrumented canal space and that from naturally occurring intraradicular moisture. The inner core of Pro Point is a mix of two proprietary nylon polymers: Trogamid $\mathrm{T}$ and Trogamid CX. The polymer coating is a cross-linked copolymer of acrylonitrile and vinylpyrroli done, which has been polymerized and cross-linked using allyl methacrylate and a thermal initiator. This nonisotropic lateral expansion is said to enhance the sealing ability of the root canal filling, thereby reducing the possibility of reinfection and potentiating the longterm success of root canal treatment.

Smartpastebio is a resin-based sealant designed to swell through the addition of ground polymer. The manufacturer claims that the addition of bioceramics gives the sealer exceptional dimensional stability and makes it nonresorbable inside the root canal. Smartpastebio produces calcium hydroxide and hydroxyapatite as by-products of the setting reaction, rendering the material both antibacterial while setting and very biocompatible once set. Also, it has a delayed setting time (4-10 hr) and is hydrophilic in nature, allowing the propoints to hydrate and swell to fill any voids. The hydrophilic nature of propoints allows the minute amount of water present in the root canal to be absorbed by the points. The rate and extent of this expansion is controlled as part of the manufacturing process. The expansion occurs within a miniscule force that claimed to be well below the reported tensile stress of dentin and a fraction of the force generated when using traditional techniques, such as warm vertical compaction. ${ }^{21,22,23}$

\section{Smart Pit And Fissure Sealants:-}

Considering the fact that occlusal surfaces constitute only $12 \%$ of the tooth surface, they are eight times as vulnerable as smooth surfaces to caries. ${ }^{24}$ So, prevention of occlusal caries assumes paramount importance in the preservation of tooth structure. The most appropriate period for the placement of occlusal sealants is soon after eruption of the permanent molars, because recently erupted teeth are less mineralized and teeth have also not undergone the benefits of post eruptive maturation of the enamel and thus more prone to acid attack. So fluoride releasing and ACP releasing pit and fissure sealants are used for this purpose. ${ }^{25}$

Fluoride releasing pit and fissure sealants - Two common methods of fluoride incorporation in to sealant are by the anion exchange system (organic fluoride compound chemically bound to the resin) and addition of fluoride salt to the unpolymerized resin. Examples are Fluoroshield and Deltonplus, contains sodium fluoride and release fluoride ions as the salt dissolves. The mechanism of fluoride release from the fluoride fissure sealant remains speculative. Fluoride release might occur from the insoluble sealant material as a result of 
porosity. It might also occur because the fluoride ion or the fluoride glass is not tightly bound to the polymerized resin molecules. Release in fluoride glass containing sealants may also be due to fluoride glass grains depositing on the surface of the resin. ${ }^{26,27,28}$

ACP releasing pit and fissure sealants- Amorphous calcium phosphate is referred to as a "smart material" because it releases calcium and phosphate ions when the surrounding $\mathrm{pH}$ drops (5.9) to a level where it could start to dissolve the tooth. Once calcium phosphate is released, it will act to neutralize the acid and buffer the $\mathrm{pH}$. ACP acts as reinforcement to the tooth's natural defence system only when it's needed. Recent decade has introduced sealants that contain fluoride and ACP. ${ }^{7,9,16,17}$

\section{Smart Dental Bonding System:-}

Now studies are trying to impart smart to dental adhesives by adding ACP, DMADDMdimethylaminododecyl methacrylate and silver nanoparticles, which make them antibacterial and self repairable. Studies have found that ACP releases calcium and phosphate ions which can remineralise tooth lesions. DMADDM and silver nanoparticles substantially decrease the biofilm and their metabolic activities and hence preventing secondary caries. ${ }^{29,30}$

\section{Smart Impression Material:-}

This new formulated material is designed with reduced contact angle, more hydrophilic to get void free impression (smart wetting). It maintains a low viscosity during working time, shape memory during elastic recovery resists distortion for more accurate impression and toughness resisting tearing of impressions. Its Snap set behaviour results in precise fitting restorations without distortion and also brings down working and setting time by at least $33 \%$. And the viscosity of these materials is very low with high flow. ${ }^{7,31}$ Ex: Imprint ${ }^{\mathrm{TM}}$ 3 VPS, Impregim ${ }^{\mathrm{TM}}$, Aquasil ultra.

\section{Smart Burs:-}

In an attempt to develop a selective caries-removal rotating instrument, a "plastic" bur was made of polyamide/imide (PAI) polymer, possessing slightly lower mechanical properties than sound dentin. Such burs have a particular hardness of $50 \mathrm{KHN}$, which is higher than the hardness of a carious dentin ( 0 to $30 \mathrm{KHN}$ ), but lower than that of sound dentin (54 to $65 \mathrm{KHN}$ ). This result in deflection and deformation of cutting blades when encountering normal or partially decalcified dentin, due to reduced cutting efficiency. Thus it removes only the caries part of the dentin (infected dentin) and the affected dentin which has the ability to remineralise is left intact. The time required for caries removal may be slightly longer, but when considered against the benefits they are amazing. ${ }^{10,32}$ Ex: SS White Smart Bur II

\section{Smart Sutures:-}

They are made up of thermoplastic polymers, having shape memory and biodegradable properties. Comprised of plastic or silk threads covered with temperature sensors and micro-heaters can detect infections. Sutures are lightly tied in its provisional shape and the ends are fixed. When the temperature is raised above the thermal transition temperature which is close to body temperature, suture would contract, thus tightening the knot with optimum force acting on the surgical sites. This is of clinical significance in tying a knot with proper stress in surgery. ${ }^{10,33}$ Ex: Novel MIT Polymer (Aachen, Germany)

\section{Smart Coatings For Dental Implants:-}

Researchers at North Carolina State University have developed a "smart coating" that helps surgical implants bond more closely with bone and ward off infection. When patients have hip, knee, or dental replacement surgery, they run the risk of having their bodies reject the implant. But the smart coating developed at NC State mitigates that risk by fostering bone growth into the implant. The coating creates a crystalline layer next to the implant and an amorphous outer layer that touches the surrounding bone. Dissolution of the amorphous layer takes place over a period of time, liberating calcium and phosphate, thus encouraging growth of the bone. "Bone grows into the coating as the amorphous layer dissolves, resulting in improved bonding, or osseointegration." Such bonding makes the implant more functional, because the bonding helps to ensure that 
the bone and the implant do a better job of sharing the load. Further development leads to incorporation of silver nanoparticles throughout the coating. These silver particles will act as antimicrobial agents and provide protection from infection at the implant site for the life of the implant. Moreover, the silver is released more quickly right after surgery, when there is more risk of infection, due to the faster dissolution of the amorphous layer of the coating. Silver release will slow down during the healing phase of the patient. That is another reason why the authors call it Smart Coating. ${ }^{34}$

\section{Smart Antimicrobial Peptide:-}

Amongst the antibiotics available, the majority affect a broad range of microorganisms, including the normal flora. The ecological disruption due to antibiotic treatment frequently results in secondary infections, super infections or other negative clinical consequences. To address this problem, recent advances has lead to the development of a new class of pathogen-selective molecules, called specific or selective targeted antimicrobial peptides (STAMPs). Example like pheromone produced by streptococcus mutans, named competence stimulating peptide(CSP) which is potent against S mutans, a cavity-causing bacterium that resides in a multispecies microbial community without affecting closely related noncariogenic oral streptococci, indicating the potential of these molecules to be developed in to "probiotic" antibiotic which could selectively eliminate pathogens while preserving the protective benefits of a healthy normal flora. Specifically targeted antimicrobial peptides (STAMP's) could be delivered in current oral care products such as mouthwash, toothpaste, or dental floss and could help with the suppression of cariogenic bacteria. ${ }^{35,36}$

\section{Smart Fibres For Laser Dentistry:-}

Laser radiation of high- fluency can be easily delivered by Hollow-core Photonic-Fibers (PCFs) i.e., the laser radiations can easily be snaked through the body using this Hollow-core Photonic-Fibers which are capable of ablating tooth enamel has been developed. These photonic fibers are known as SMART FIBRES. 40 ps of laser pulses with a total energy up to $2 \mathrm{~mJ}$ coupled into a Hollow core of a Photonic Crystal Fibre with a core diameter of approximately $14 \mu \mathrm{m}$ are focused on a tooth surface to induce an optical breakdown, resulting in plasma formation and dental tissue ablation. The laser breakdown was visualized as optical characterization of the ablated enamel surface. Emission from laser produced plasmas transmitted through the Hollow core PCF in the backward direction and analysed with a Monochromator and a CCD camera.

Thus, Photonic Crystal Fiber is not only to transport the high power laser pulse to a tooth surface, but also to transmit plasma emission to the system for detection and optical diagnosis. One should be very cautious while using these fibers because there are chances of fiber wall failure, there may be spurt of laser light, thus causing hazard for the healthy tissue. ${ }^{37,38}$

\section{Conclusion:-}

The recent advances in the design of smart materials have created novel opportunities for their application in dental field which is an indicator of potential and rapid progress in this area. This class of materials has the potential to execute specific functions intelligently in order to anticipate challenges based on the potential to recognize, analyze, and discriminate the local environment changes. The most sophisticated class of smart materials in the foreseeable future will be that which emulates the biological system. Thus these innovations in material science have marked the beginning of an era in biosmart dentistry, a step into the future.

\section{References:-}

1. JF McCabe, Z Yan, OT Al Naimi, G Mahmoud, SL Rolland. Smart materials in dentistry. Australian Dental Journal 2011;56:(1 Suppl): 3-10.

2. Mangaiyarkarasi S P , Manigandan T, Sivagami S, Rajendran Vijayakumar, Nagarjuna Chowdary. Biosmart Dentistry. Annals and Essences of Dentistry 2013;4: 34-38.

3. Smart materials and systems. Post note. (Parliamentary Office Of Science and Technolog, UK) 2008;299:1-4.

4. Gil FJ, Planell JA. Shape memory alloys for medical applications. Proc Inst Mech Eng H 1998;212:47388. 
5. Lendlein A, Langer R. Biodegradable, elastic shape-memory polymers for potential biomedical applications. Science 2002;296:1673-6.

6. Stayton PS, El-Sayed ME, Murthy N, Bulmus V, Lackey C, Cheung C et al. 'Smart' delivery systems for biomolecular therapeutics. Orthodontics Craniofacial Res 2005;8:219-25.

7. M. Shanthi, E.V. Soma Sekhar, Swetha Ankireddy. Smart materials in dentistry: Think smart! Journal of Pediatric Dentistry $2014 ; 1: 1-4$.

8. Rolland SL, McCabe JF, Robinson C, Walls AW. In vitro biofilm formation on the surface of resin-based dentine adhesives. Eur J Oral Sci 2006;114:243-9.

9. Dube M, Ponnappa KC. Smart ART! J Int Oral Health 2009;1:52-7.

10. Padmawar N, Pawar N, Joshi S, Mopagar V, Pendyala G, Vadvadgt V. Biosmart Dental Materials: A New Era in Dentistry. Int J Oral health med res 2016;3(1):171-176.

11. McCabe JF, Carrick TE, Sidhu SK. Determining low levels of fluoride released from resin based dental materials. European journal of oral sciences 2002;110:380-384.

12. Itota T, Carrick TE, Yoshiyama M, McCabe JF. Fluoride release and recharge in giomer, compomer and resin composite. Dent Mater 2004;20:789-795.

13. J.R .Kelly, "Dental ceramics: what is this stuff any way?" Journal of the American Dental Association $2008 ; 139: 4 \mathrm{~S}-7 \mathrm{~S}$.

14. J.R. Kelly and I. Denry, "Stabilized zirconia as a structural ceramic: an overview," Dental Materials 2008;24:289-298

15. J.R. Kelly and P. Benetti, "Ceramic materials in dentistry: historical evolution and current practice," Australian Dental Journal 2011;56:84-96

16. D.Skrtic and J.M.Antonucci, "Bioactive polymeric composites for tooth mineral regeneration: physicochemical and cellular aspects," Journal of Functional Biomaterial 2011;2:271-307

17. Jie Zhao, Yu Liu, Wei-bin Sun and Hai Zhang, "Amorphous calcium phosphate and its application in dentistry," Chemistry Central Journal 2011;5(40)

18. K.D. Jandt and B.W. Sigusch, "Future perspectives of resin based dental materials," Dental Materials 2009;25(8):1001- 1006

19. R.S.Trask, H.R.Williams and I.P.Bond, "Self-healing polymer composites: mimicking nature to enhance performance," Bioinspiration and Biomimetics 2007;2(1):1-9

20. E.C.Teixeira,S.C.Bayne,J.Y.Thompson,A.V.Ritter and E.J.Swift, "Shear bond strength of self etching bonding systemsin combination with various composites used for repairing aged composites," Journal of Adhesive Dentistry 2005;7(2):159-164

21. Didato A, Eid AA, Levin MD, Khan S, TayFR, RueggebergFA. Time-based lateral hygroscopic expansion of water-expandable endodontic obturation point. J Dent 2013 ;41(9):796-801.

22. D.J. Highgate and J. D. Frankland, "Deformable polymeric compositions," US patent no 1986;4:565

23. D.J.Highgate and J.A.Lloyd, "Expandable/contractable composition for surgical or dental use," US patent no 2007;7(210):935

24. Charbeneau GT, Dennison J. Clinical success and potential failure after single application of a pit and fissure sealant: Four-year report. J Am Dent Assoc 1979;98:559-564.

25. Tonia L. Morphis, Jack Toumba. K and Nick A. Lygidakis. Fluoride pit and fissure sealants: A review. International Journal of Paediatric Dentistry 2000;10:90-98.

26. Lygidakis NA, Oulis KI. A comparison of Fluroshield with Delton fissure sealant: Four year results. Pediatr Dent. 1999; 21:429-431.

27. Richard J. Simonsen. Pit and fissure sealant: Review of the literature. Pediatr Dent. 2002;24:393-414.

28. Pit and fissure sealant with ACP products restorative aegis ${ }^{\circledR}$ pit \& fissure sealant with ACP. Harry J. Bosworth Company, 2002-2013.

29. Chen Chena, Michael D. Weir, Lei Cheng, Nancy J. Lin, Sheng Lin-Gibson, Laurence C. Chow, Xuedong Zhou, Hockin H.K. Xu. Antibacterial activity and ion release of bonding agent containing amorphous calcium phosphate nano particles. Dental materials .2014;30: 891-901

30. Mary Anne S. Melo, Lei Cheng, Ke Zhang, Michael D. Weir, Lidiany K.A. Rodrigues, Hockin H.K. Xu. Novel dental adhesives containing nanoparticles of silver and amorphous calcium phosphate. Dental materials .2013;29:199-210 
31. Terry DA, Leinfelder KF, Lee EA, James A. The impression: A blue print to restorative success. Int Dent SA 2006;8:12-21.

32. Dammaschke T, Rodenberg TN, Schäfer E, Ott KH. Efficiency of the polymer bur SmartPrep compared with conventional tungsten carbide bud bur in dentin caries excavation. Oper Dent 2006;31:256-60.

33. Patel P. Smart sutures that detect infections. Bio Med News 2012.

34. X. Bai, K. More, C. M. Rouleau, and A. Rabiei, "Functionally graded hydroxyapatite coatings doped with antibacterial components," Acta Biomaterialia 2010;6(6):2264-2273

35. Eckert R, He J, Yarbrough DK, Qi F, Anderson MH, Shi W. Targeted killing of Streptococcus mutans by a pheromone-guided —-smart antimicrobial peptide. Antimicrobial Agents Chemotherapy 2006;50(365):1 7.

36. Alexander Asokan, Haalaswamy V Kambalimath, Raju Umaji Patil, KP Bharath. Stamps: A goodbye message to oral pathogens!. Indian Journal of Oral Sciences 2015;6(1):1-6.

37. Konorov SO, Mitrokhin VP, Fedotov AB, SidorovBiryikov DA, Beloglazov VI, Skibina NB et al. Hollowcore photonic-crystal fibres for laser dentistry. Phys. Med. Biol 2004;49(7):1359-1368.

38. Mahavir.B.Mishra, Shanu Mishra . Lasers And Its Clinical Applications In Dentistry. International Journal Of Dental Clinics 2011;3(4):35-38. 